\title{
Role of Mini-Percutaneous Nephrolithotomy in the Treatment of Renal Stones
}

\author{
Irfanullah, Zahid ullah khan, Muhammad Asad shamsher, Muhammad Izhar*, Riaz Ahmad \\ Khan, Bakhtawar Gul \\ Institute of Kidney Diseases, Peshawar, Pakistan. \\ *Corresponding Author: Muhammad Izhar, Institute of Kidney Diseases, Peshawar, Pakistan.
}

\section{Abstract}

Objective: To determine the role of mini-percutaneus nephrolithotomy in the renal stones management and its post-operative complications.

Methodology: This descriptive study was conducted at Institute of Kidney Diseases (IKD), Hayatabad Medical Complex, Peshawar from January 2017 to January 2019 over 154 patients. Baseline investigations were carried out for all the patients fulfilling inclusion criteria. All patients provided written, informed consent before the procedure. Demography, operative parameters, and outcomes were analyzed in the study. X-ray KUB was done postoperatively for all patients and follow-up ultrasound examination/NCCT at follow-up visit at 1 month.

Results: Out of 154 patients, 90(58.44\%) were male and 64(41.55\%) were female. Mean age of the subjects were $33.3 \pm \mathbf{8 . 5}$ (15-70) years. 84(54.54\%) patients had left renal stones while $\mathbf{7 0 ( 4 5 . 4 5 \% ) ~ p a t i e n t s ~ h a d ~ r i g h t ~}$

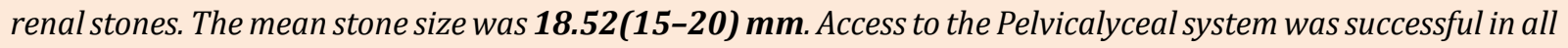
(100\%) cases. The mean operation time was 53.14(45-60) minutes. The mean hospital stay was 2(2-3) days. The targeted calyces were upper in 10(6.49\%), middle in $\mathbf{9 0}(\mathbf{5 8 . 4 4 \% )}$, and lower in $\mathbf{5 4 ( 3 5 . 0 6 \% )}$ patients. Complete Stone clearance was achieved in 140(90.90\%) patients while 14(9.09\%) patients had residual stone fragments, out of which 2 patients underwent ureteroscopic fragmentation while 4 patients required post op extracorporeal shock wave lithotripsy (ESWL) for the residual stones. No major complications occurred.

Conclusion: Mini- percutaneus nephrolithotomy is an effective minimally invasive modality of treatment in renal stones and is associated with excellent stone clearance, less morbidity and shorter hospital stay

Keywords: Percutaneous nephrolithotomy, mini-PCNL, Renal stones.

\section{INTRODUCTION}

Humans have been affected by urinary stone disease for centuries. Different treatment options are available for the management of renal stones, depending upon the bulk and location of the stones. The management of stone disease has been changed.The first case of the removal of renal stones by means of nephrostomy by Rupel and Brown $^{1}$ in 1941.Percutaneous nephrolithotomy (PCNL) is a minimally invasive surgical modality, was first performed in 1976 by Fernastrom and Johansson, for the management of renal stones. ${ }^{2,3}$ In comparison to other management modalilities mini-PCNL has advantage of high stone clearance rate ${ }^{4}$ Based on the statistics reported by
British Association of Urological Surgeons (BAUS) ${ }^{5}$ and Clinical Research Office of the Endourological Society (CROES) ${ }^{6}$, risks associated with PCNL consist of postoperative sepsis (2\%), fever (10\%-16\%), and injury to adjacentorgans $(0.4 \%)$. In significantbleeding $(8 \%)$ are common complications after this procedure , with potentially dangerous consequences.

Keeping in view the recognized risks of hemorrhage and organ injury associated with tract dilation in conventional PCNL, a key strategy is designed at, by reducing the tract size by the miniaturization of equipment/instrument size, as well as the use of laser technology and enhanced optic systems. There has been a dramatic shift in the indications of PCNL 
over the last decade. Some studies indicated that by reducing the tract size, complications associated with percutaneous surgery might be reduced ${ }^{7}$. After the introduction of miniaturized instruments including mini-PCNL, ultra mini-PCNL and micro-PCNL, there is no need to dilate the tract over $20 \mathrm{Fr}$. Jackman et al. ${ }^{8}$ first introduced minimally invasive PCNL (mini-PCNL) for pediatric age patients in 1998.

In 2001, a particularly designed miniaturized nephroscope for mini-PCNL in adults was first introduced by Lahme et al. ${ }^{9}$ in Germany,after which the procedure of "mini-PCNL" gained popularity rapidly and became more and more admired worldwide. The first generation minimally invasive PCNL (MIP) system had a number of key features which comprise single-stage dilatation of the tract. This also includes additional method of stone clearance by the "vacuum cleaner" effect ${ }^{\mathbf{1 0}}$. Since then, mini-PCNL has gained popularity worldwide.

In 2010 Schilling et al. ${ }^{11}$ reported that this practice had completely replaced conventional PCNL in their unit. In terms of improved stone clearance and complications Mini-PCNL is more successful than retrograde intrarenal surgery (RIRS) ${ }^{12}$ for patients with solitary kidney having stones with size larger than $2 \mathrm{~cm}$, in patients having congenitally deformed kidneys such as horseshoe kidney, polycystic kidney, and transplanted kidney ${ }^{13}$.

The aim of this study is to evaluate the role of miniPCNL particularly focusing on stone clearance, complications, and retreatment rate.

\section{Methodology}

This was a prospective observational study. Which includes 154 consecutive patients who underwent mini-PCNL from January 2017 to January 2019. The inclusion criteria includes all patients having age 14 years and above, with renal stones having size 15$20 \mathrm{~mm}$ in diameter. Subjects with blood dyscrasias ,renal anatomic anomalies and pregnant females are excluded from the study. Detailed history and physical examination was done followed by routine baseline investigations included full blood counts, renal function tests, electrolytes, bleeding parameters, and urine culture. Patient with active urinary tract infection were treated before the procedure with intravenous antibiotics according to $\mathrm{C} / \mathrm{S}$ report, till urine culture became negative. Stone burden was evaluated using a multi-slice helical computed tomography (CT) scan. All patients were provided written, informed consent before the procedure. Demography, operative parameters, and outcomes were analyzed in the study. All patients had postoperative plain X-ray KUB before discharge from the hospital, and follow-up ultrasound examination/NCCT at follow-up visit at 1 month.

\section{Surgical Technique}

The whole min-PCNL procedure was performed under general anesthesia. Intravenous prophylactic antibiotics were administrated preoperatively. Csytoscopy performed and then 6 Fr open ended ureteral catheter passed into the ureter. Urinary bladder was catheterized with 16 Fr Foley's catheter and the patient was turned prone. The initial puncture was obtained using fluoroscopic guidance by gradual descent technique over the desired calyx with 18gauge needle and a glide wire was introduced. Initially with use of facial dilator $10 \mathrm{~F}$ dilatation was performed. Then further dilatation was performed with the single step metal dilator. Stones were broken using a $550 \mu \mathrm{m}$ holmium stone laser fiber or by ballistic lithotripsy. Stone pieces were removed by the "vacuum cleaner effect". Adherent fragments were extracted with stone grasper. At the end of the procedure, a JJ stent was placed antegradely or the ureteric catheter was left overnight for drainage. Nephrostomy tube was placed in some case while others were tubeless. Once the stone was cleared, the clearance was confirmed with direct nephroscopy and fluoroscopy. If complete stone clearance was not achievable with single access tract, a second access using a $17 \mathrm{~F}$ Amplatz sheath was placed under fluoroscopic control. In cases of remaining large fragments, at the end of the procedure, a $12 \mathrm{~F}$ nephrostomy tube was placed to allow for a secondlook PCNL 7-10 days later.

Postoperatively, all patients were given standard intravenousantibioticsandnarcoticanalgesicsregimen. X-Ray KUB were done on the 1st post-operative day to look for any residual stone in te pelvicalyceal system. In case of no second look procedure, nephrostomy was removed.JJ stents were removed after 2 weeks. Patients were discharged on $1^{\text {st }}$ or $2^{\text {nd }}$ postoperative day. Complication occured intraoperatively or within 30 days were graded by the modified Clavien-Dindo classification system ${ }^{14}$.Stone $\geq 5 \mathrm{~mm}$ was considered as clinically significant residual stone fragment (RSF). Stone asymptomatic and size $<5 \mathrm{~mm}$ was termed as 
Role of Mini-Percutaneous Nephrolithotomy in the Treatment of Renal Stones

clinically insignificant residual stone (CIRF). Complete stone clearance is considered when no residual stone is noted on conventional X-ray. Follow-up of all the patients were carried out at one and three months. Plain X-Ray KUB for radiopaque stones, ultrasound abdomen \& pelvis for radiolucent stones , urinalysis and serum creatinine, were performed.

\section{RESUlts}

\section{Patient and Stone Demographics}

In all, 154 patients underwent mini-PCNL.Mean age of the sample was $33.3 \pm 8.5$ (15-70) years. In all, $84(54.54 \%)$ patients had stones on left-side while 70 (45.45\%) patients had right-sided renal stones. The mean stone size was $18.52(15-20) \mathrm{mm}$. Stones were located in upper calyx in $10(6.49 \%)$, middle calyx $30(19.48 \%)$, lower calyx in 60 (38.96\%), the renal pelvis in $50(32.46 \%)$ patients, and upper ureter $4(2.59 \%)$. Upper ureteric stones were pushed into renal pelvis during ureteric catheterization.

Patient and Stone Demographics

\begin{tabular}{|l|l|}
\hline Variables & Value \\
\hline Number of patients & 154 \\
\hline Gender & $\mathrm{N}(\%)$ \\
Male & $90(58.44 \%)$ \\
Female & $64(41.55 \%)$ \\
\hline Age & $33.3 \pm 8.5(15-70)$ years \\
\hline Side & $\mathrm{N}(\%)$ \\
Left & $84(54.54 \%)$ \\
Right & $70(45.45 \%)$ \\
\hline Stone size, mm & $18.52(15-20) \mathrm{mm}$ \\
\hline Access to pelvicalyceal system & $\mathrm{N}(\%)$ \\
Upper-calyx & $10(06.49 \%)$ \\
Middle-calyx & $30(19.48 \%)$ \\
Lower-calyx & $60(38.96 \%)$ \\
Renal-pelvis & $50(32.46 \%)$ \\
Upper-ureter & $04(02.59 \%)$ \\
\hline
\end{tabular}

\section{Access and Instruments}

In all, 154(100\%) mini-PCNL cases were performed. Access to pelvicalyceal system was achieved through a single puncture in 130 (84.41\%) cases, while in $24(15.58 \%)$ patients with double puncture. Lower calyceal approach in 54 (35.06\%) cases, middle calyx 90 (58.44\%), and upper calyx 10(6.49\%). Mini PCNL access sheath of $17.5 \mathrm{~F}$ was used in all cases. Glide wire were used in all cases during initial puncture.
Access and Instruments

\begin{tabular}{|c|l|}
\hline $\begin{array}{c}\text { Position } \\
\text { Prone } \\
\text { Supine }\end{array}$ & $154(100 \%)$ \\
\hline $\begin{array}{c}\text { Punctures } \\
\text { Single }\end{array}$ & $130(84.41 \%)$ \\
Double & $24(15.58 \%)$ \\
\hline Access & $10(06.49 \%)$ \\
Upper calyx & $90(58.44 \%)$ \\
Middle calyx & $54(35.06 \%)$ \\
Lower calyx & $17.5 \mathrm{~F}$ \\
\hline Access sheath &
\end{tabular}

\section{Fragmentation and Stones Removal}

The lithoclast used was pneumatic to break stones in $110(71.42 \%)$ cases and a holmium laser in 44 (28.57\%) cases. Stone fragments were retrieved using forced irrigation from ureteric catheter in $104(67.53 \%)$ cases and graspers in 50(32.46\%) cases. The mean duration for procedure was 53.14(45-60) min. The operative duration was related to stone burden. $140(90.90 \%)$ patients had Complete stone clearance while $14(9.09 \%)$ patients had residual stones. Out of which 2 patient required ureterorenoscopic stone fragmentation (URS) and 4 patients required post-operative ESWL while in the rest 8 patients stone fragments were so small that they passed spontaneously during the follow up. Post operatively drainage was provided in the form of a nephrostomy tube and JJ stent in $44(28.57 \%)$ cases while in $100(64.93 \%)$ cases tubeless procedures (only JJ stent) were perfumed. In 10(6.49\%) cases were total tubeless procedures were performed. The mean inpatient stay was 2 (2-3) days.

\section{Operative Details}

\begin{tabular}{|l|l|}
\hline Fragmentation: & $\mathrm{N}(\%)$ \\
Pneumatic lithoclast & $110(71.42 \%)$ \\
Holmium laser & $44(28.57 \%)$ \\
\hline $\begin{array}{l}\text { Stone extractors: } \\
\text { Forced irrigation } \\
\text { Gracepers }\end{array}$ & $104(67.53 \%)$ \\
\hline Operative time & $50(32.46 \%)$ \\
\hline Stone clearance rate: & $53.14(45-60)$ min. \\
Complete & $140(90.90 \%)$ \\
Residual fragments & $14(09.09 \%)$ \\
\hline Drainage: & $44(28.57 \%)$ \\
Nephrostomy+JJ & $100(64.93 \%)$ \\
Tubeless (only JJ) & $10(6.49 \%)$ \\
Total tubeless & $2(2-3)$ days \\
\hline Hospital stay & \\
\hline
\end{tabular}

Archives of Urology V4 . I1 . 2021 
Role of Mini-Percutaneous Nephrolithotomy in the Treatment of Renal Stones

\section{Complications}

Complications were classified according to Clavien Dindo classification. No serious complications were seen during the study. Complications occurred in $39(25.32 \%)$ cases. Of them $19(12.33 \%)$ were Clavien Grade 1, 11(7.14\%) were Clavien Grade 2, and 9(5.84\%) were Grade 3 (5.84\%). There were no Grade 4 or 5 complications.

Grade 1 complications like, Transient fever (more than $\left.38^{\circ} \mathrm{C}\right)$ in $14(9.09 \%)$ and transient elevation of creatinine occurred in 5(3.29\%) patients which responded to the usual treatment.

Grade 2 complications, perinephrostomy leakage $6(3.89 \%)$ patients, blood transfusion in $2(1.2 \%)$ patients and pneumonia occurred in $3(1.94 \%)$ patients, which responded to usual treatment.

Grade 3 complication, like urinary leakage occurred in $3(1.94 \%)$ patients which were managed with JJ stent placement. 2 patients required URS and 4 patients required post-operative ESWL for residual stones.

\begin{tabular}{|c|l|}
\hline $\begin{array}{c}\text { Complications } \\
\text { Yes }\end{array}$ & \multicolumn{1}{|c|}{$\mathrm{N}(\%)$} \\
No & $115(25.32)$ \\
\hline Grade -1 complications & $19(12.33 \%)$ \\
Grade -2 complications & $11(07.14 \%)$ \\
Grade -3 complications & $09(5.84 \%)$ \\
Grade -4 complications & $00(00)$ \\
Grade -5 complications & $00(00)$ \\
\hline
\end{tabular}

\section{DisCUSSION}

Percutaneous nephrolithotomy is recommended nowdays as procedure of choice for stones measuring $>2 \mathrm{~cm}$ in diameter with high stone free rate. ${ }^{15}$ Due to its high safety and efficacy it os highly demanding procedure nowdays. ${ }^{16}$. in Standard PCNL access to the pelvicalyceal system is carried out by using amplatz sheath of 24-30 F while mini-PCNL is performed by using amplatz sheath upto $18 \mathrm{~F}$ with less bleeding compication ${ }^{17,18}$. Further, to decrease the morbidity and mortiliy of this procedure, minimally invasive techniques are established. Amplatz sheath of $18 \mathrm{Fr}$, one step dilator and low prsssure irrigation is used. Stone fragments are retrieved without any use of forcep.

Although mini-PCNL results in complete stone free rate in $92.9 \%$ of patients with kidney stone of $<2 \mathrm{~cm}^{19}$.
However,it also give good results in patients with a larger stone size. Limitations may be the conversion of mini-PCNL to conventional PCNL where required.

In the present study, we have achieved excellent stone clearance rate of $90.90 \%$, which is comparable to internationally reported outcomes of mini-PCNL. These results are supported by Li et al. $^{20}$ who had stone free rate in his study $89 \%$ for his 4760 mini-PCNL. Similarly, Zeng et al. ${ }^{21}$ had $90 \%(146)$ stone free rate for his super mini percutaneous nephrolithelopexy. While Abdelhafez et al. $^{22}$ and Agrawal et al. ${ }^{23}$ had 84\%(191) and 99\%(120) stone free rates. Our results are consistent with international studies in terms of stone clearance.

Jackman et al. ${ }^{17}$ reported, SFR of $89 \%$ with mean hospital stay of 1.7 days.They concluded that miniPCNL may be benifical in comparison to conventional PCNL for per-op bleedings risk, post-op hospital stay, and postoperative analgesia, which confirms our present results.

Operative time for our study was 53.14(45-60) min and blood transfusion in $2(1.2 \%)$ patients. Yang et al. ${ }^{24}$ reported Stone free rate of $97.2 \%$ with mean operative time of $45 \mathrm{~min}$ for patients with proximal ureteric stones. Decreased transfusion rate in miniPCNL may be due to less trauma to parenchyma and large segmental vessels by suing small bore amplatz sheath. The decreased operative time might be due to "vacuum cleaner effect" in which stone fragments are retrieved without using forcep. Retrieval of small fragments occurs when turbulence flow occurs at the tip of sheath with fragments finding low pressure area to escape. The process of fragmentation in miniCNL can be done with laser and ballistic lithotripsy. High-frequency and low-energy 'dust' settings are preferable ${ }^{25}$. In our procedure, stne fragmentation was done with pneumatic lithoclast and holmium laser.

The fragmentation of stone with the mini PCNL is possible with ballistic lithotripsy and laser. We used both pneumatic lithoclast and holmium laser for stone fragmentation.

According to British Association of Urological Surgeons (BAUS) PCNL registry and CROES PCNL registry, overall complication rate was $21.3 \%$ and it was $20.5 \%$ respectively ${ }^{5,26}$. Our complications rate was consistent with international studies and were primarily low grade in nature. No Clavien IV/V complications were recorded. 
Role of Mini-Percutaneous Nephrolithotomy in the Treatment of Renal Stones

\section{Conclusion}

Mini-PCNL is an effective minimal invasive management modality in renal stones and is associated with excellent stone clearance, less morbidity and shorter hospital stays.

\section{REFERENCES}

[1] Rupel E, Brown R. Nephroscopy with removal of stone following nephrostomy for obstructive calculus anuria. J Urol 1941; 46:177-82.

[2] Fernstrom I, Johansson B. Percutaneous pyelolithotomy: a new extraction technique. Scand J UrolNephrol 1976; 10:257-9.

[3] Vicentini FC, Gomes CM, Danilovic A, Neto EA, Mazzucchi E, Srougi M. Percutaneous nephrolithotomy: current concepts.Indian J Urol 2009; 25:4-10.

[4] Zhu W, Liu Y, Liu L, Lei M, Yuan J, Wan SP, et al. Minimally invasive versus standard percutaneous nephrolithotomy: a meta-analysis. Urolithiasis2015; 43:563-70.

[5] Armitage JN, Irving SO, Burgess NA, British Association of urological Surgeons Section of Endourology. Percutaneous nephrolithotomy in the United Kingdom: results of a prospective data registry. EurUrol2012; 61:1188-93.

[6] de la Rosette J, Assimos D, Desai M, Gutierrez J, Lingeman J, Scarpa R, et al. The Clinical Research Office of the Endourological Society percutaneous nephrolithotomy global study: indications, complications, and outcomes in 5803 patients. J Endourol2011; 25:11-7

[7] Ganpule AP, Bhattu AS, Desai M. PCNL in the twenty-first century: role of microperc, miniperc, and ultraminiperc. World J Urol 2015; 33: 235240 .

[8] Jackman SV, Hedican SP, Peters CA, Docimo SG. Percutaneous nephrolithotomy in infants and preschool age children: experience with a new technique. Urology 1998; 52:697-701.

[9] Lahme S, Bichler KH, Strohmaier WL, Gotz T. Minimally invasive PCNL in patients with renal pelvic and calyceal stones. EurUrol2001; 40:61924.

[10] Nicklas AP, Schilling D, Bader MJ et al. The vacuum cleaner effect in minimally invasive percutaneous nephrolitholapaxy. World J Urol 2015; 33: 1847-53

[11] Schilling D, Gakis G, Walcher U, Stenzl A, Nagele U. The learning curve in minimally invasive percutaneous nephrolitholapaxy: a 1-year retrospective evaluation of a novice and an expert.World J Urol2011; 29: 749 - 53

[12] Zeng G, Zhu W, Li J, Zhao Z, Zeng T, Liu C, et al. The comparison of minimally invasive percutaneous nephrolithotomy and retrograde intrarenal surgery for stones larger than $2 \mathrm{~cm}$ in patients with a solitary kidney: a matched-pair analysis. World J Urol2015; 33:1159-64.

[13] Wu W, Zhao Z, Zhu H, Yang D, Ou L, Liang Y, et al. Safety and efficacy of minimally invasive percutaneous nephrolithotomy in treatment of calculi in horseshoe kidneys. J Endourol 2014; 28:926-9.

[14] Tuerk C, Knoll T, Petrik A, Sarica K, Straub M, Seitz C. EAU Guidelines on Urolithiasis. Arnhem, The Netherlands: European Association of Urology, updated March 2011. Available at: www.uroweb. org/gls/pockets/english/20\%20 Urolithiasis. pdf. Accessed March 2012

[15] Michel MS, Trojan L, Rassweiler JJ. Complications in percutaneous nephrolithotomy.EurUrol2007; 51: $899-906$

[16] Jackman SV, Docimo SG, Cadeddu JA, Bishoff JT, KavoussiLR,JarrettTW.The'mini-perc'technique: a less invasive alternative to percutaneous nephrolithotomy. World J Urol1998; 16: 371 - 4

[17] Knoll T, Wezel F, Michel MS, Honeck P, WendtNordahl G. Do patients benefit from miniaturized tubeless percutaneous nephrolithotomy? A comparative prospective study. J Endourol 2010; 24: $1075-9$

[18] Nagele U, Schilling D, Anastasiadis AG et al. Minimally invasive percutaneous nephrolitholapaxy (MIP).Urologe A 2008; 47: 1066, $8-73$

[19] Li X, He Z, Wu K et al. Chinese minimally invasive percutaneous nephrolithotomy: The Guangzhou experience. J Endourol 2009; 23: 1693-7 
Role of Mini-Percutaneous Nephrolithotomy in the Treatment of Renal Stones

[20] Zeng G, Wan S, Zhao Z et al. Super-mini percutaneous nephrolithotomy (SMP): a new concept in technique and instrumentation. BJU Int 2016; 117: 655-61

[21] Abdelhafez MF, Amend B, Bedke J et al. Minimally invasive percutaneous nephrolithotomy: a comparative study of the management of small and large renal stones. Urology 2013; 81: 241-5

[22] Agrawal MS, Agarwal K, Jindal T, Sharma M. Ultra-mini-percutaneous nephrolithotomy: a minimally-invasive option for percutaneous stone removal. Indian J Urol 2016; 32: 132-6
[23] Yang SF, Lei M, Li X. Minimally invasive percutaneous nephrolithotomy for impacted upper ureteric calculi (a report of 71 cases). Chin J Mod Operative Surg2003; 4:297-8.

[24] Bader MJ, Pongratz T, Khoder W et al. Impact of pulse duration on Ho: YAG laser lithotripsy: fragmentation and dusting performance. World J Urol 2015; 33: 471-7

[25] Kamphuis GM, Baard J, Westendarp M, de la Rosette JJ. Lessons learned from the CROES percutaneous nephrolithotomy global study. World J Urol.2015;33(2):223-33.

Citation: Irfanullah, Muhammad Izhar, et al. Role of Mini-Percutaneous Nephrolithotomy in the Treatment of Renal Stones. Archives of Urology. 2021; 4(1): 09-14. DOI: https://doi.org/10.22259/2638-5228.0401003

Copyright: (C) 2021 Irfanullah, Muhammad Izhar, et al. This is an open access article distributed under the Creative Commons Attribution License, which permits unrestricted use, distribution, and reproduction in any medium, provided the original work is properly cited. 